\title{
Tau regulates the subcellular localization of calmodulin
}

\author{
Elena Gómez de Barreda a , Jesús Avila a,b,* \\ a Centro de Biología Molecular “Severo Ochoa”, CSIC/UAM, Universidad Autónoma de Madrid, Cantoblanco, 28049 Madrid, Spain \\ ${ }^{\mathrm{b}}$ CIBER de Enfermedades Neurodegenerativas, 28031 Madrid, Spain
}

\section{A R T I C L E I N F O}

\section{Article history:}

Received 13 April 2011

Available online 21 April 2011

\section{Keywords:}

Tau

Calmodulin

Calbindin

\begin{abstract}
A B S T R A C T
Lack of tau expression in neuronal cells results in a change in the expression of few genes. However, little is known about how tau regulates gene expression. Here we show that the presence of tau could alter the subcellular localization of calmodulin, a protein that could be located at the cytoplasm or in the nucleus. Nuclear calmodulin binds to co-transcription factors, regulating the expression of genes like calbindin. In this work, we have found that in neurons containing tau, a higher proportion of calmodulin is present in the cytoplasm compared with neurons lacking tau and that an increase in cytoplasmic calmodulin correlates with a higher expression of calbindin.
\end{abstract}

(c) 2011 Elsevier Inc. All rights reserved.

\section{Introduction}

Tau is a microtubule-associated protein (MAP) that is mainly expressed in neurons [1], where tau is mainly found associated to polymerized tubulin, a cytoplasmic protein that is the major component of brain microtubules. However, tau could be also present free in the cytoplasm [2] or bound to other proteins. Several tau-associated proteins have been described [1], being one of them calmodulin [3-5]. Calmodulin (CaM) is a major calcium sensor in neurons [6] when is present in the cytoplasm. Also, it can bind and regulate diverse target proteins by different mechanisms [7]. In the nucleus, calmodulin binds to some co-transcription factors, like BAF-57 [8], a protein member of a complex involved in the repression of neuronal specific genes [9]. Little is known about how the subcellular localization of calmodulin is regulated, a process that, by it self, could regulate calmodulin functions. In neuronal cells it has been indicated that depolarization could induce the elevation of nuclear CaM in dentate gyrus granule cells and that nuclear CaM accumulation depends on the association of CaM with an endogenous CaM binding protein [10]. In other cases, for specific isoforms of calmodulin, posttranslational modifications of the protein could influence its subcellular localization [11].

Recently, it has been shown that tau protein could regulate the expression of few genes, mainly related to neuron phenotype [12].

\footnotetext{
Abbreviations: MAP, microtubule associated protein; CaM, calmodulin; wt, (wild type); BSA, bovine serum albumin; PBS, phosphate buffered saline.

* Corresponding author at: Centro de Biología Molecular "Severo Ochoa", CSIC/ UAM, Fac. Ciencias, Universidad Autónoma de Madrid, Cantoblanco, 28049 Madrid, Spain. Fax: +34 911964420

E-mail address: javila@cbm.uam.es (J. Avila).
}

However, nothing is known about the mechanism for that regulation for specific genes.

Since tau is mainly a cytoplasmic protein, we have studied if the binding of calmodulin to tau could result in retaining calmodulin at the cytoplasm and if it results in a decrease in the nuclear function of calmodulin, a function that could be related with the expression of neuronal genes like calbindin [8].

In this work, we have found that the lack of tau in neurons changes the subcellular localization of calmodulin and that it correlates with a change in the expression of calbindin.

\section{Methods}

\subsection{Antibodies}

The following primary antibodies were used: anti-calmodulin (Millipore); anti-calbindin (Chemicon); anti- $\beta$-III-tubulin (Covance); anti- $\beta$-actin (Sigma Aldrich); anti-tubulin (Sigma Aldrich); anti-lamin B1 (Santa Cruz Biotechnology). The 7.51 antibody (from Dr. Claude Wischik, UK) was used to detect tau in a phosphorylation-independent manner [13] and detects all soluble tau isoforms in Western blot analysis.

\subsection{Animals}

$\mathrm{Tau}^{-1-}$ mice were generated as previously described [14] and can be obtained from Charles Rivers Co. Heterozygous $\left(\mathrm{Tau}^{+/-}\right.$) mice were crossed in order to obtain homozygous tau knockout mice (Tau) and control littermates $\left(\mathrm{Tau}^{+/+}\right)$. Animals were bred at the Centro de Biología Molecular "Severo Ochoa" (Madrid, Spain). Four to five mice were housed per cage with food and water avail- 
A
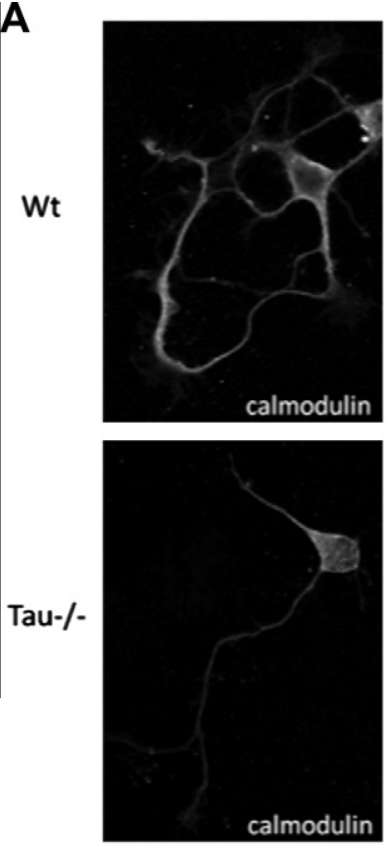
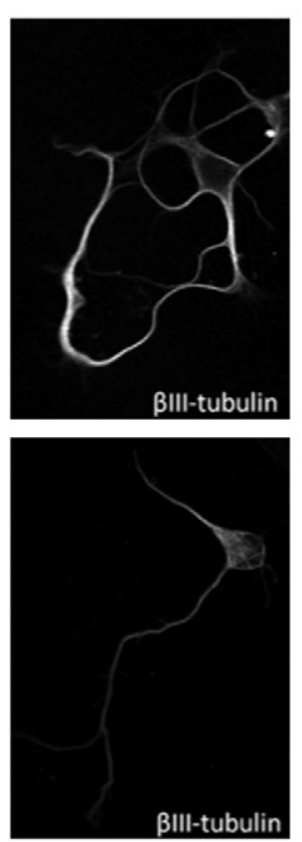
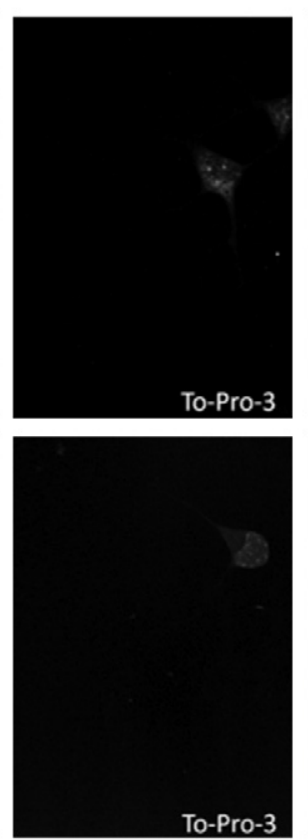
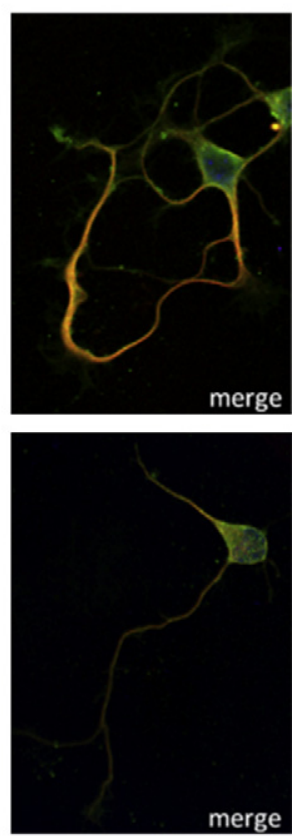

B

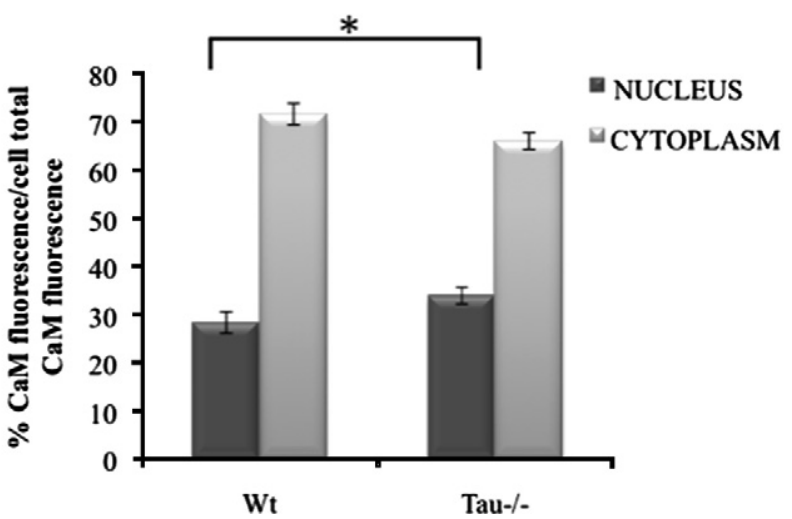

C

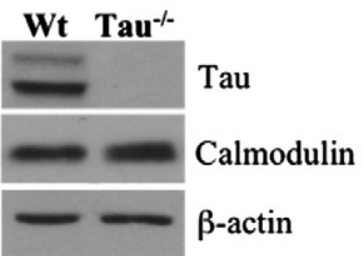

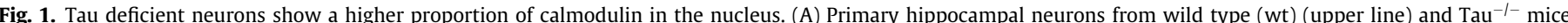

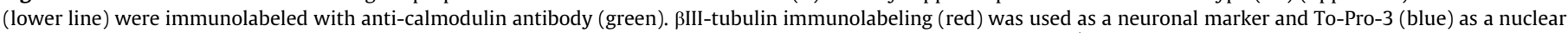

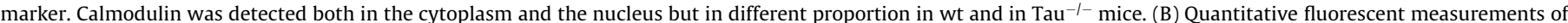

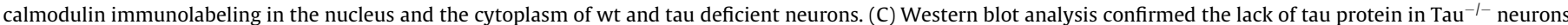

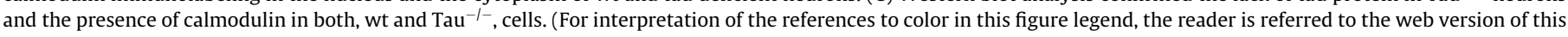
article.)

able ad libitum. Mice were maintained in a temperature-controlled environment on a 12/12 h light-dark cycle with light onset at 07:00 h. All experiments were carried out with males.

\subsection{Cell cultures}

Primary cultures of hippocampal neurons were prepared according to established procedures with slight modifications [15]. Briefly, hippocampal tissue was obtained from E18 mouse embryos, dissected, and dissociated with the Papain Dissociation System (PDS; Worthington Biochemical Corporation, Lakewood, NJ, USA). Neurons were maintained in Neurobasal medium (Gibco, Grand Island, NY, USA) supplemented with $2 \%$ B-27, 1\% N2, $0.5 \mathrm{mM}$ glutamine, $100 \mathrm{U} / \mathrm{ml}$ penicillin, and $100 \mathrm{mg} / \mathrm{mL}$ streptomycin, and grown on poly-L-lysine $(100 \mathrm{mg} / \mathrm{mL})$ coated plastic dishes. The cells were incubated in $95 \%$ air $/ 5 \% \mathrm{CO}_{2}$ in a humidified incubator at $37^{\circ} \mathrm{C}$.

Human neuroblastoma SH-SY5Y cells [16] were maintained in Dulbecco's modified Eagle's medium (DMEM) supplemented with $10 \%$ fetal bovine serum, $2 \mathrm{mM}$ glutamine, $100 \mathrm{U} / \mathrm{ml}$ penicillin,
$100 \mathrm{U} / \mathrm{ml}$ streptomycin in a humified atmosphere of $5 \% \mathrm{CO}_{2} / 95 \%$ air at $37^{\circ} \mathrm{C}$. For differentiation, proliferating SH-SY5Y cells were plated and then cultured in Neurobasal-B27 medium (Gibco, Grand Island, NY) supplemented with $2 \mathrm{mM}$ dibutyril cyclic AMP and $1 \mathrm{mM}$ glutamine for 7 days.

\subsection{Immunofluorescence}

Cells were permeabilized in $0.1 \%$ Triton $\mathrm{X}-100$ for $15 \mathrm{~min}$ and then they were treated with $1 \mathrm{M}$ glycine for $30 \mathrm{~min}$ to eliminate their auto fluorescence. After a brief wash with PBS, they were incubated with $1 \%$ bovine serum albumin (BSA)/phosphate buffered saline (PBS) for $1 \mathrm{~h}$ and then exposed to primary antibody diluted in $1 \% \mathrm{BSA} / \mathrm{PBS}$ for $1 \mathrm{~h}$. Primary antibodies anti- $\beta$-III-tubulin (1:2000; Covance) and anti-calmodulin (1:200; Millipore) were used. Cultures were extensively washed and then incubated with the appropriate secondary antibody, conjugated either with Alexa 488 or with Alexa 555 (1:200; Invitrogen), plus To-Pro-3 (1:500; Invitrogen) (nucleic acid stain) for $45 \mathrm{~min}$. After washing, 
A
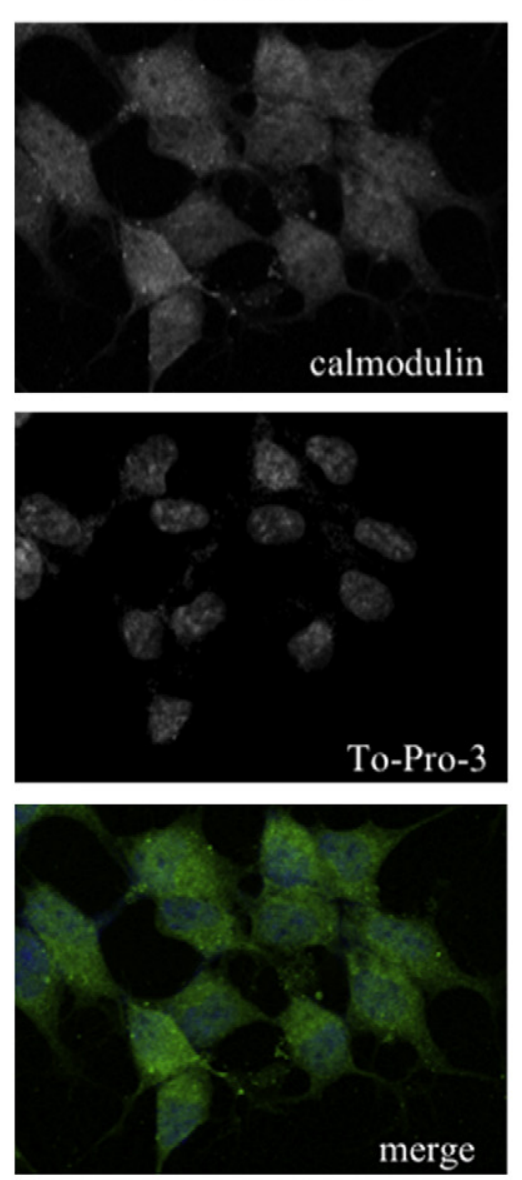

B

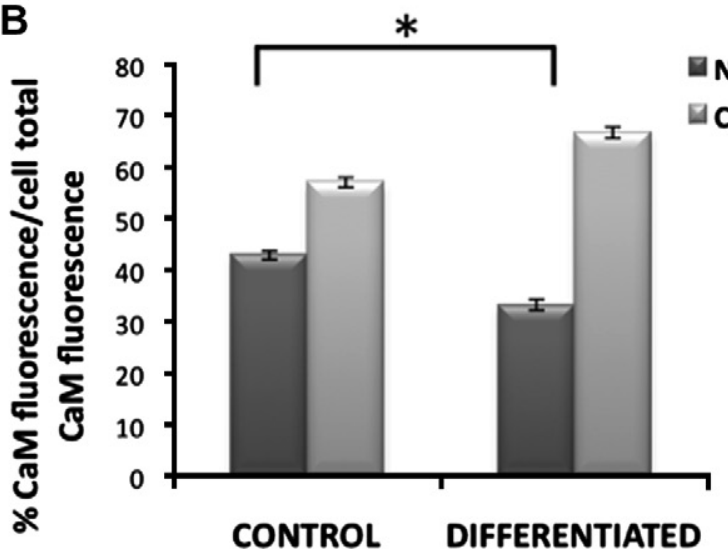

CONTROL
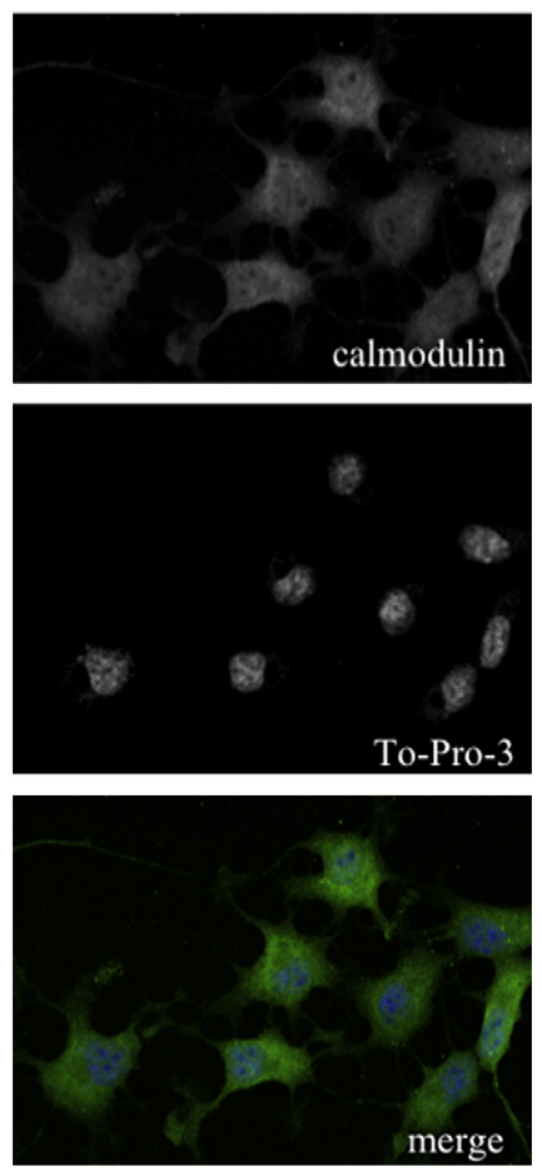

C

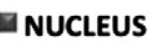

G CYTOPLASM

C DIFF

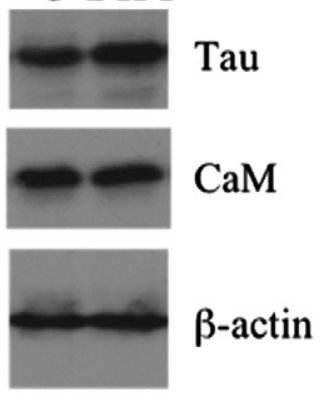

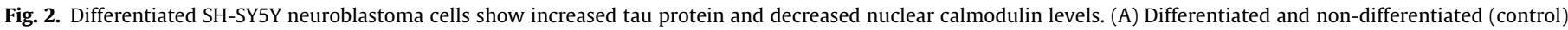

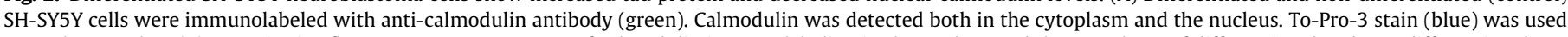

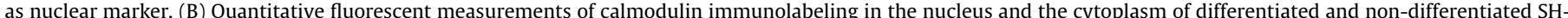

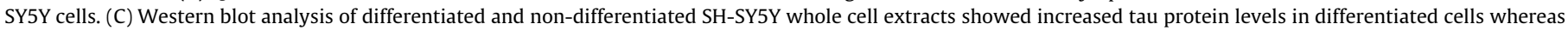
total calmodulin levels were similar. (For interpretation of the references to color in this figure legend, the reader is referred to the web version of this article.)

they were immediately mounted with Fluorosave Reagent (Calbiochem).

\subsection{Western blot analysis}

Cells were lysed in buffer consisting of 20 mM HEPES, pH 7.4, $100 \mathrm{mM} \mathrm{NaCl}, 20 \mathrm{mM} \mathrm{NaF}, 1 \%$ Triton X-100, 1 mM sodium orthovanadate, $5 \mathrm{mM}$ EDTA, and $2 \mathrm{mM}$ PMSF and a protease inhibitor cocktail (Roche). Samples were electrophoresed on 12\% SDS-poly- acrylamide gel and transferred to a nitrocellulose membrane (Schleicher \& Schuell, Keene, NH). The experiments were performed using the following primary antibodies: 7.51 anti-tau (1:100), anti-calmodulin (1:1000), anti- $\beta$-actin (1:5000), anti-lamin $B(1: 200)$, anti- $\alpha$-tubulin $(1: 5000)$ and anti-calbindin $(1: 1000)$. The filters were incubated with the antibody at $4{ }^{\circ} \mathrm{C}$ overnight in $5 \%$ non-fat dried milk. Secondary goat anti-mouse (1:1000) (Dako), goat anti-rabbit $(1: 1000)$ (Dako) and donkey anti-goat $(1: 1000)$ (Santa Cruz Biotechnology) antibodies and ECL detection reagents 

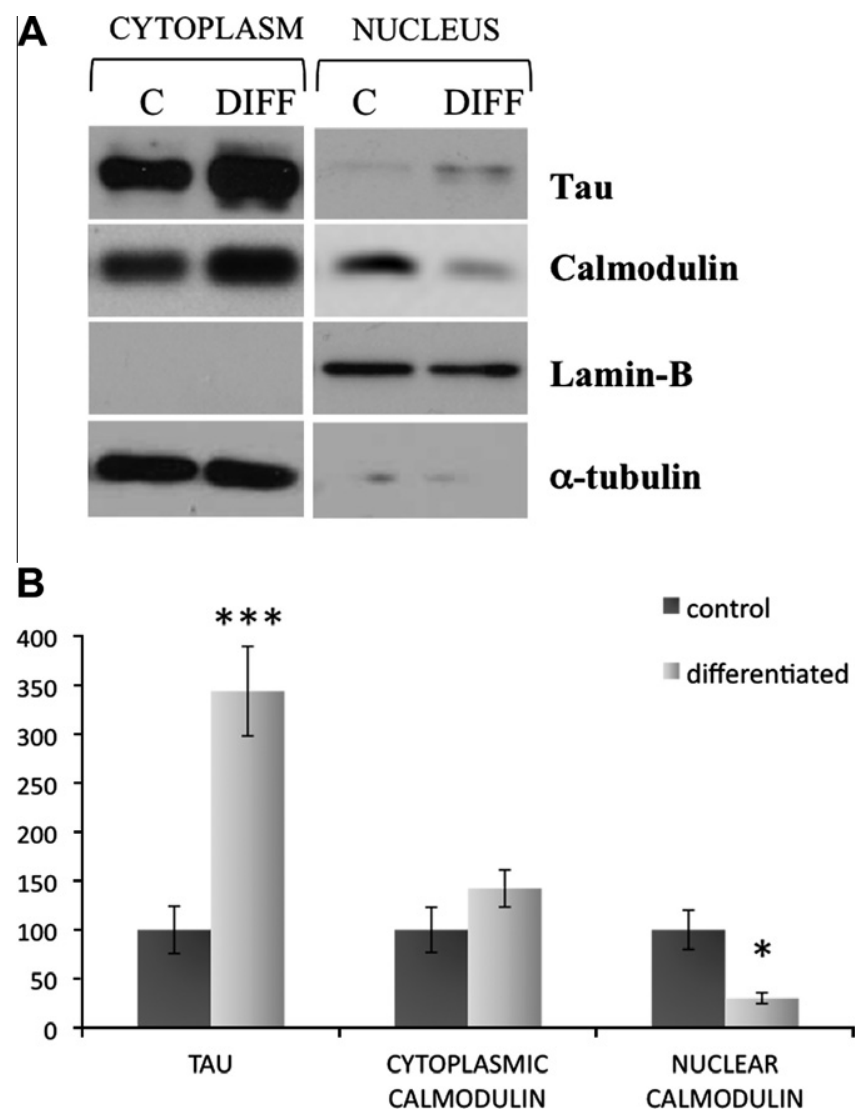

Fig. 3. Differentiated neuroblastoma SH-SY5Y cells show decreased nuclear calmodulin levels. (A) Cytoplasmic and nuclear fractions from differentiated and non-differentiated (control) SH-SY5Y cells were analyzed by Western blotting to detect calmodulin and tau. Lamin-B was used as nuclear load marker and $\alpha$-tubulin as cytoplasmic load marker. (B) Protein quantization in cytoplasm and nucleus of differentiated and non-differentiated cells $\left({ }^{* * *} p<0005 ;{ }^{*} p<0,05\right)$.
(Amersham Biosciences, Arlington Heights, IL) were used for immunodetection.

\subsection{Subcellular fractionation}

Cells were collected in M-SHE buffer ( $210 \mathrm{mM}$ manitol, $70 \mathrm{mM}$ sucrose, 10 mM HEPES pH 7.4, 1 mM EDTA, 1 mM EGTA) supplemented with protease inhibitors ( $1 \mathrm{mM}$ phenylmethylsulfonyl fluoride, $10 \mu \mathrm{g} / \mathrm{ml}$ aprotinin, $10 \mu \mathrm{g} / \mathrm{ml}$ leupeptin, $10 \mu \mathrm{g} / \mathrm{ml}$ pepstatin), phosphatase inhibitors ( $1 \mathrm{mM}$ sodium orthovanadate, $30 \mathrm{mM}$ glycerol phosphate, $5 \mathrm{mM}$ pyrophosphate, $1 \mu \mathrm{M}$ okadaic acid) and $1 \mathrm{mM}$ dithiothreitol. Cytoplasmic and nuclear fractions were extracted as previously described [12]. Lysis buffer (50 mM TrisHCI pH 6.8, 10 mM $\beta$-mercaptoethanol, 2\% SDS, 0.1\% bromophenol blue, $10 \%$ glycerol) was added to both fractions. Samples were boiled for $10 \mathrm{~min}$ and then analyzed by SDS-PAGE.

\subsection{RNA isolation and quantitative real-time $P C R$}

RNA was isolated from differentiated and non-differentiated SH-SY5Y cells using the Absolutely RNA MiniPrep Kit (Stratagene). This RNA was used as a template for the first strand cDNA synthesis (Roche), which was then analyzed by quantitative real-time PCR with an Applied Biosystems ABI PRISM 7900HT real-time detection system with the SYBR green PCR master mix (Applied Biosystems), interpreting the results with the SDS 2.1 software. The primers used to amplify each gene were: 5'-ACAGCATGTCTACTGCT TGGATCCA-3' and 5'-TGGTGCTCCCTTCCCTTGTC ACA-3' for human calbindin; 5'-CACACTGTGCCCATCTACGA-3' and 5'-CTCCTTAATGTCACGCACGA-3' for human $\beta$-actin.

\section{Results}

In previous studies it was reported a direct binding between tau and calmodulin [3-5]. Since tau is mainly a cytoplasmic protein, its interaction with calmodulin could affect to the subcellular localization of calmodulin. Thus, we have tested that possibility.
A

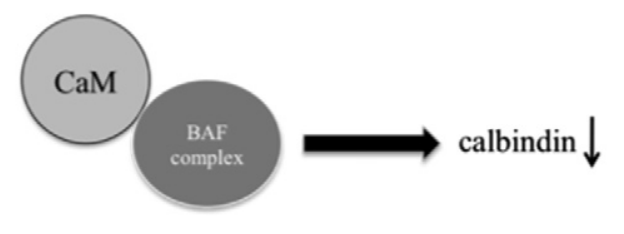

B

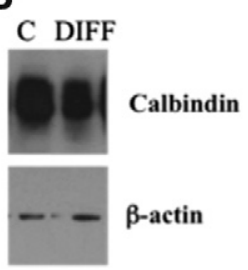

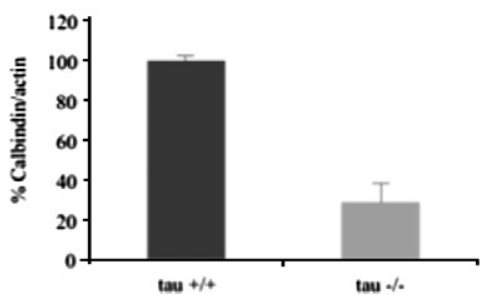

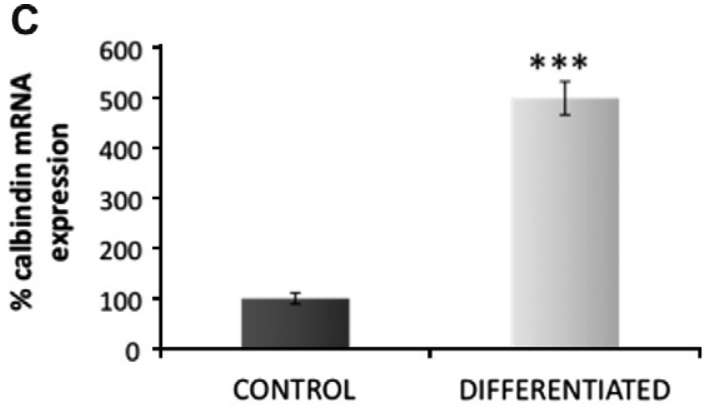

D

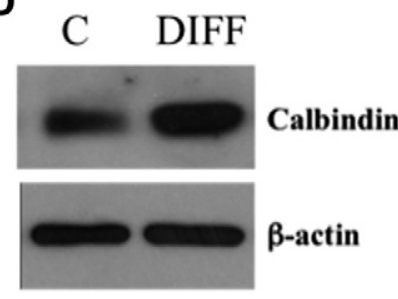

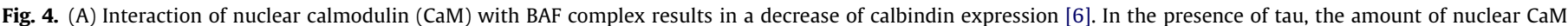

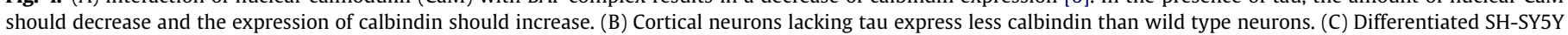

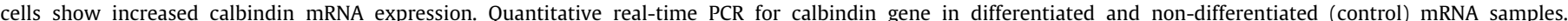

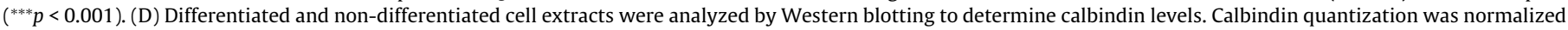
to $\beta$-actin levels $\left({ }^{*} p<0.05\right)(\mathrm{E})$. 


\subsection{The presence of tau increases cytoplasmic localization of calmodulin}

By comparing hippocampal neuron cultures from wild type mouse or from a mouse lacking tau we found, by immunofluorescence analysis, increased levels of nuclear calmodulin in neurons from $\mathrm{Tau}^{-1-}$ mice (Fig. 1A) compared to wild type mice. The presence of total tau and calmodulin was quantitated in both wild type and $\mathrm{Tau}^{-1-}$ neurons (Fig. 1B). As expected a dramatic difference was found in the amount of tau from both mice but not in the amount of calmodulin (Fig. 1C).

A similar result was found when undifferentiated and differentiated human neuroblastoma SY5Y cells were tested. In undifferentiated cells a lower tau level was found compared with differentiated cells as determined by Western blot analyses (Fig. 2C, see also Fig. 3A), whereas no changes in the amount of calmodulin were observed (Fig. 2). Again, a higher nuclear localization for calmodulin was found in non-differentiated cells compared to differentiated neuroblastoma cells (Fig. 2A and B).

\subsection{Quantization of calmodulin in the cytoplasm and cell nucleus}

To test, biochemically, the subcellular localization of calmodulin, subcellular fractionation to obtain cytoplasmic and nuclear fractions was performed, followed by Western blot analysis to identify calmodulin and tau in those fractions. Fig. 3 shows an increase in nuclear calmodulin in cells lacking tau. Thus, the presence of tau correlates with a decrease in calmodulin localization at the cell nucleus.

\subsection{In the absence of tau there is an increase in calbindin expression}

A described function for nuclear calmodulin is its interaction with transcriptional factors $[8,10]$ being one of them BAF57, a protein of the BAF complex. Upon binding of calmodulin, the function of BAF complex is activated [6] and the expression of neuronal proteins like calbindin is decreased [12,17] (Fig. 4A). Thus, we have tested if a decrease in the amount of nuclear calmodulin, due to the absence of tau could result in an increase in calbindin expression. First, we tested the expression in cortical neurons containing or lacking tau. Fig. 4B shows an increase in calbindin expression in neurons containing tau protein. Also, we observed that differentiated SY5Y cells, with higher tau expression than undifferentiated cells, showed an increase in calbindin expression (Fig. 4A and B), as determined by calbindin mRNA and protein (Fig. 4C and D).

\section{Discussion}

In a previous report [12], we showed that tau deficiency lead to the upregulation of BAF57, a protein that is involved in down regulation of neuron-specific gene expression [6]. In addition, in this work, we have found that tau deficiency also regulates calbindin expression. It can take place by activation of BAF complex through its interaction with nuclear calmodulin, as previously reported [8].

Indeed, in previous studies it has been described that nuclear accumulation of calmodulin could be due to its interaction with some nuclear proteins [10]. Now we suggest that cytoplasmic accumulation of calmodulin could be due to its interaction with tau protein.

Thus, our results suggest that tau protein may regulate the subcellular localization and function of calmodulin in neurons. Tau protein binds to calmodulin through an ionic interaction in a sim- ilar fashion to the binding between tau and other calcium binding proteins like tubulin $[18,19]$. After that interaction, the presence of calmodulin at the cell nucleus decreases, probably due to the fact that tau protein is mainly a cytoplasmic protein and that the complex tau-calmodulin should be mainly located at the cytoplasm. In the absence of tau or the presence of low tau levels, the level of calmodulin at the cell nucleus increases. Nuclear calmodulin could act as a co-transcription factor regulating the expression of neuronal proteins, like calbindin, facilitating the process of neuron differentiation, in which tau expression may play a role [12,14].

\section{Acknowledgments}

This work was supported by Grants from Spanish Plan Nacional, Comunidad de Madrid, CIBERNED and Fundación M. Botín.

We would like to acknowledge the skillful technical assistance of Ms. L. Teresa.

\section{References}

[1] J. Avila, J.J. Lucas, M. Perez, F. Hernandez, Role of tau protein in both physiological and pathological conditions, Physiol. Rev. 84 (2004) 361-384.

[2] B. Esmaeli-Azad, J.H. McCarty, S.C. Feinstein, Sense and antisense transfection analysis of tau function: tau influences net microtubule assembly, neurite outgrowth and neuritic stability, J. Cell Sci. 107 (Pt 4) (1994) 869-879.

[3] Y.C. Lee, J. Wolff, Calmodulin binds to both microtubule-associated protein 2 and tau proteins, J. Biol. Chem. 259 (1984) 1226-1230.

[4] K. Sobue, M. Fujita, Y. Muramoto, S. Kakiuchi, The calmodulin-binding protein in microtubules is tau factor, FEBS Lett. 132 (1981) 137-140.

[5] R. Padilla, R.B. Maccioni, J. Avila, Calmodulin binds to a tubulin binding site of the microtubule-associated protein tau, Mol. Cell Biochem. 97 (1990) 35-41.

[6] K.P. Hoeflich, M. Ikura, Calmodulin in action: diversity in target recognition and activation mechanisms, Cell 108 (2002) 739-742.

[7] W.Y. Cheung, Calmodulin plays a pivotal role in cellular regulation, Science 207 (1980) 19-27.

[8] D. Lai, M. Wan, J. Wu, P. Preston-Hurlburt, R. Kushwaha, T. Grundstrom, A.N. Imbalzano, T. Chi, Induction of TLR4-target genes entails calcium/calmodulindependent regulation of chromatin remodeling, Proc. Natl. Acad. Sci. USA 106 (2009) 1169-1174.

[9] E. Battaglioli, M.E. Andres, D.W. Rose, J.G. Chenoweth, M.G. Rosenfeld, M.E. Anderson, G. Mandel, REST repression of neuronal genes requires components of the hSWI.SNF complex, J. Biol. Chem. 277 (2002) 41038-41045.

[10] P.G. Mermelstein, K. Deisseroth, N. Dasgupta, A.L. Isaksen, R.W. Tsien, Calmodulin priming: nuclear translocation of a calmodulin complex and the memory of prior neuronal activity, Proc. Natl. Acad. Sci. USA 98 (2001) 15342 15347.

[11] A. Dong, H. Xin, Y. Yu, C. Sun, K. Cao, W.H. Shen, The subcellular localization of an unusual rice calmodulin isoform, OsCaM61, depends on its prenylation status, Plant Mol. Biol. 48 (2002) 203-210.

[12] E. Gomez de Barreda, H.N. Dawson, M.P. Vitek, J. Avila, Tau deficiency leads to the upregulation of BAF-57, a protein involved in neuron-specific gene repression, FEBS Lett. 584 (2010) 2265-2270.

[13] M. Novak, R. Jakes, P.C. Edwards, C. Milstein, C.M. Wischik, Difference between the tau protein of Alzheimer paired helical filament core and normal tau revealed by epitope analysis of monoclonal antibodies 423 and 7.51, Proc. Natl. Acad. Sci. USA 88 (1991) 5837-5841

[14] H.N. Dawson, A. Ferreira, M.V. Eyster, N. Ghoshal, L.I. Binder, M.P. Vitek, Inhibition of neuronal maturation in primary hippocampal neurons from tau deficient mice, J. Cell Sci. 114 (2001) 1179-1187.

[15] F. Hernandez, M. Perez, J.J. Lucas, A.M. Mata, R. Bhat, J. Avila, Glycogen synthase kinase-3 plays a crucial role in tau exon 10 splicing and intranuclear distribution of SC35. Implications for Alzheimer's disease, J. Biol. Chem. 279 (2004) 3801-3806.

[16] J.L. Biedler, S. Roffler-Tarlov, M. Schachner, L.S. Freedman, Multiple neurotransmitter synthesis by human neuroblastoma cell lines and clones, Cancer Res. 38 (1978) 3751-3757.

[17] N. Ballas, C. Grunseich, D.D. Lu, J.C. Speh, G. Mandel, REST and its corepressors mediate plasticity of neuronal gene chromatin throughout neurogenesis, Cell 121 (2005) 645-657.

[18] L. Serrano, A. Valencia, R. Caballero, J. Avila, Localization of the high affinity calcium-binding site on tubulin molecule, J. Biol. Chem. 261 (1986) 70767081.

[19] L. Serrano, J. de la Torre, R.B. Maccioni, J. Avila, Involvement of the carboxylterminal domain of tubulin in the regulation of its assembly, Proc. Natl. Acad. Sci. USA 81 (1984) 5989-5993. 